\title{
Herpes Zoster Anterior Uveitis with Hyphema
}

\author{
Rajagopal Arvinth, Mimiwati Zahari, and Sagili Chandrasekhara Reddy
}

\section{ABSTRACT}

Herpes zoster ophthalmicus usually manifests in elderly people as a unilateral painful skin rash in a dermatomal distribution of the ophthalmic division of the trigeminal nerve shared by the eye and ocular adnexa. It is the reactivation of varicella-zoster infection. Most common ocular manifestations of this disease include keratitis, conjunctivitis, and anterior uveitis. Severe hyphema as a complication following herpes zoster uveitis is uncommon. We report a rare case of a 90-year-old lady with herpes zoster ophthalmicus and diabetes mellitus with unusually severe hyphema. The disease responded well to systemic and topical steroids with improved vision outcome. Early referral to an ophthalmologist, detailed evaluation, and immediate treatment are mandatory to prevent permanent vision loss in these patients.

Keywords: Anterior uveitis, herpes zoster ophthalmicus, hyphema, raised intraocular pressure.

Submitted : August 10, 2021
Published : September 3, 2021
ISSN: 2593-8339
DOI: 10.24018 /ejmed.2021.3.5.1010
R. Arvinth
Department of Ophthalmology, Faculty
of Medicine, University of Malaya,
Malaysia. Ophthalmology,
Department of
Hospital Ampang, Malaysia.
M. Zahari
Department of Ophthalmology, Faculty
of Medicine, University of Malaya,
Malaysia
S. C. Reddy*
Department of Ophthalmology, Faculty
of Medicine and Defence Health,
National Defence University of
Malaysia, Malaysia.
(e-mail: profscreddy gmail.com)
*Corresponding Author

herpes zoster ophthalmicus with iridocyclitis and hyphema in a very elderly ( 90 years old) lady with diabetes mellitus, in view of its rare occurrence.

\section{CASE Report}

A 90-year-old lady, diabetic for more than 20 years with previous good health, presented to the eye clinic of our hospital with painful right macular rash over the forehead on the right side, root of the nose, and redness of right eye for the past one week which was worsening day by day (Fig. 1). On presentation, vision in the affected right eye was counting finger at 2 meters. Anterior segment examination of right eye with slit lamp showed diffuse congestion of the conjunctiva, multiple punctate epithelial erosions, keratic precipitates (Fig. 2), and flare and cells in the anterior chamber. Pupil was circular and reacting sluggishly to light. Lens examination showed nuclear sclerosis cataract. Applanation tonometry showed intraocular pressure (IOP) of $15 \mathrm{mmHg}$. Dilated pupil fundoscopy revealed moderate non-proliferative diabetic retinopathy. Hutchinson's sign was positive. The left eye had a vision of 6/36, with nuclear sclerosis cataract. Intraocular pressure was $18 \mathrm{~mm} \mathrm{Hg}$. Fundoscopy showed mild nonproliferative diabetic retinopathy.

We diagnosed her as having herpes zoster ophthalmicus with anterior uveitis and the patient was admitted in the eye ward. Blood investigations (full blood count, erythrocyte 
sedimentation rate and C-reactive protein) revealed no abnormalities. Fasting blood sugar was within normal range with already taking diabetic medication Tab. Metformin 1 gram two times daily.

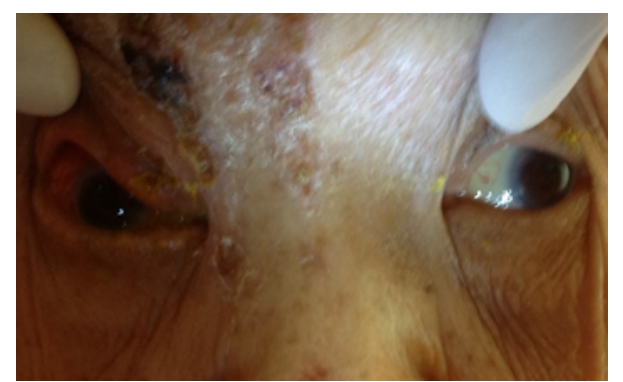

Fig. 1. Showing skin rash along the ophthalmic division of the trigeminal nerve on the right side of forehead, root of the nose, and congestion of right eye.

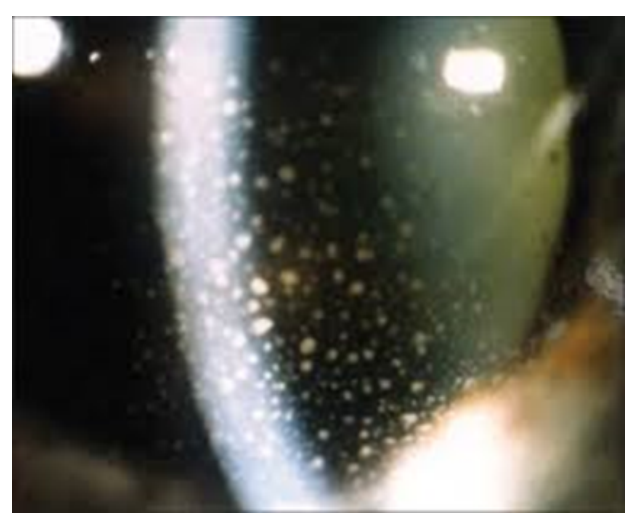

Fig. 2. Slitlamp photograph (magnified view) of the cornea showing circular multiple keratic precipitates (cellular deposits on the corneal endothelium) and nuclear sclerosis of the lens.

Dexamethasone eye drops $(0.1 \%) 4$ hourly, homatropine eye drops (2\%) 3 times, Acyclovir eye ointment (3\%) 5 times daily in the right eye and oral Acyclovir $800 \mathrm{mg} 5$ times a day for one week were started. After 24 hours, patient went home against the medical advice on the same treatment. However, three days after that, patient came back to the eye clinic with history of sudden loss of vision in right eye. There was no history of fall, head trauma or any injury sustained to the eye.

Right eye examination: Vision worsened to perception of light. Keratic precipitates were present. Severe hyphemia (two-thirds of anterior chamber covering the upper margin of the pupil) was noted (Fig. 3). Intraocular pressure was 24 $\mathrm{mmHg}$. Ultrasound B- scan did not show any vitritis, or vitreous haemorrhage, or retinal detachment.

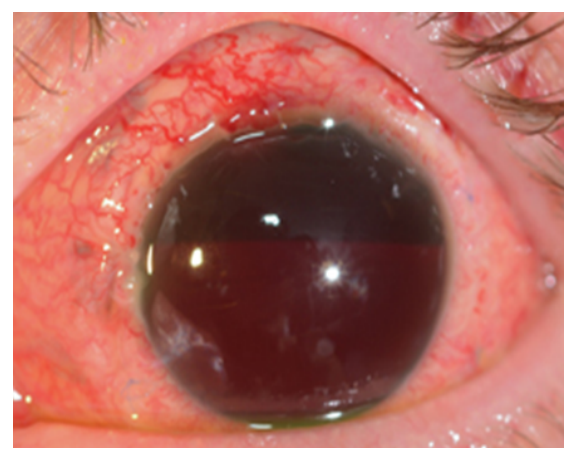

Fig. 3. Showing blood in anterior chamber (hyphema) covering upper margin of the pupil.
In addition to topical treatment, Tab. Acetazolamide $250 \mathrm{mg}$ QID, Tab. Prednisolone 10mg QID were started, and Gutt Timolol $0.5 \%$ BD was added in right eye to reduce the IOP. After one week of treatment, vision improved to $6 / 60$, hyphema level became less (blood up to $1 / 3$ of anterior chamber) and the intraocular pressure reduced to $17 \mathrm{mmHg}$. Tab. Acetazolamide and Tab. Prednisolone were reduced to 3 times daily. During the follow up visits after one-week, anterior segment examination showed very minimal hyphema, and presence of segmental iris atrophy (thin areas on the iris) from 3 to 7 o'clock. No rubeosis (new blood vessels on the iris) was noted on the iris. Intraocular pressure in the right eye was $16 \mathrm{mmHg}$.

The frequency of topical treatment in the right eye was reduced (homatropine eye drops once daily, dexamethasone eye drops two times daily acyclovir eye ointment three times daily); and timolol eye drops were continued two times daily. Tab. Acetazolamide was reduced to bid for 2 days and then stopped; Tab. Prednisolone was also tapered at three days interval in one week time. An anterior chamber paracentesis to demonstrate the presence of the varicella-zoster virus in the aqueous fluid (polymerase chain reaction test) was planned. However, we were unable to get the test done because the patient did not consent for the procedure, and she was not financially able to pay the cost of the test. The patient was asked to come for follow up after one week. Unfortunately, she defaulted the follow up.

\section{DISCUSSION}

Herpetic anterior uveitis is the most common cause of viral anterior uveitis accounting for $5-10 \%$ of all uveitis cases in the western world [12] and $0.9-8.3 \%$ of all infectious uveitis in India [13]. Varying prevalence of anterior uveitis has been reported in $17.8 \%$ [14], $29.4 \%$ [15], and $46.6 \%$ [1] of herpes zoster ophthalmicus patients.

Ocular involvement in HZO may be varied in the form of macular/pustular rash over the eyelids, conjunctivitis (congestion and edema of conjunctiva), punctate epithelial keratitis and the hallmark pseudodendrites with negative fluorescein staining i.e. fluorescein collects at the edges of the epithelium rather than staining an epithelial defect, anterior stromal keratitis (nummular keratitis - multiple small circular white opacities in the cornea), disciform stromal keratitis (involving endothelium) resulting in corneal opacification, raised intraocular pressure due to trabeculitis, anterior uveitis (iritis, iridocyclitis), episcleritis/scleritis, acute retinal necrosis (rapid necrotic inflammation of the retina resulting in retinal detachment in $50 \%$ of patients and often permanent vision loss) [1]. Rarely, ocular cranial nerve palsy and optic neuritis are also reported in HZO patients [14], [15].

Anterior uveitis typically results in an anterior chamber cellular reaction and can progress to synechiae formation with adhesion of the iris to either the lens or the angle structures inside the anterior chamber, and sectoral iris atrophy. Hyphema in cases of herpes zoster anterior uveitis is relatively uncommon [4], [5], [7], [9]. Tugal-Tutkun et al. [8] did not find any hyphemia in 111 cases of herpes zoster induced anterior uveitis; however, they reported distorted pupil, posterior synechiae, elevated IOP, sectoial iris atrophy 
in these patients. In cases of herpes zoster uveitis with hyphema, it is widely believed that the presenting hyphema is caused by occlusive vasculitis to the iris leading to ischemia and bleeding into anterior chamber [2], [5], [6]. Severe iris atrophy seen after resolution of the hyphema suggests the possibility of severe inflammation that may have caused the obstruction of iris vessels. Hyphema showed a tremendous reduction upon starting prednisolone [7], and similar observation was noted in our patient also.

An iris fluorescein angiography might help to rule out rubeosis as a cause of the hyphema [4]. In view of severe hyphema, it was not performed in our patient. In an aqueous-based polymerase chain reaction (PCR) study of viral anterior uveitis cases, Babu et al from South India, reported $66.6 \%$ of cases were varicella zoster virus (VZV), $19.4 \%$ were herpes simplex virus (HSV-1), and $8.3 \%$ were cytomegalo virus (CMV) [13]. An anterior chamber paracentesis yielding a positive result of viral DNA can help in the definitive diagnosis of varicella zoster virus (VZV) causing the herpes zoster uveitis [16]. In cases whereby the viral DNA PCR yields a negative result, a progressive decrease in serial anti-VZV antibody measurement, can strongly indicate the virus as a causative agent [7]. However, the gold standard investigation is still the detection of the virus via DNA PCR. In our patient the above investigations were not done due to financial constraints.

\section{CONCLUSION}

Occurrence of hyphema in herpes zoster anterior uveitis is uncommon. Early referral of herpes zoster ophthalmicus patents to the ophthalmologist is vital to detect and give appropriate treatment for eye lesions to save the vision. Presence of hyphemia in iridocyclitis is an indication of herpes zoster aetiogy in a patient with cutaneous lesions.

\section{ETHICAL APPROVAL}

It is not applicable.

\section{CONSENT}

The patient was admitted in the eye ward. Informed consent was taken for investigations and further management.

\section{REFERENCES}

[1] B. P. Yawn, P. C. Wollan, J. L. StSauver, L. C. Butterfield, "Herpes zoster eye complications: rates and trends," Mayo Clinic Protocol, 88(6):562-70, 2013.

[2] S. Saad, T. A. Christopher, "Evaluation and Management of Herpes Zoster Ophthalmicus,” Am Fam Physician, 66(9):1723-1730, 2002.

[3] J. Frary, P. T. Petersen, M. Pareek, "Hutchinson's sign of ophthalmic zoster," Clin Case Rep, 8(1):219-220, 2020.

[4] S. Hayasaka, M. Watanabe, Y. Yamamoto, S. Noda, M. Sekimoto, T. Setogawa, "Herpes Zoster Opthalmicus complicated by hyphema and haemorrhagic glaucoma," Ophthalmologica, 196(4):185-187, 1988.

[5] E. K. Akpek, J. D. Gottsch, "Herpes zoster sine herpete presenting with hyphema," OcularImmunol Inflamm, 8(2): 115-118, 2000.

[6] K. Babu, V. K. Konana, S. K. Ganesh, G. Patnaik, N. S. Chan, S. P. Chee, ... and M. Zierhut, "Viral anterior uveitis," Indian J Ophthalmol, 68:1764-73, 2020.
[7] Y. Okunuki, J. Sakai, T. Kezuka, H. Goto, "A Case of Herpes Zoster Uveitis with severe hyphema," BMC Ophthalmology, 14: 74-77, 2014.

[8] I. Tugal-Tutkun, B. Otük-Yasar, E. Altinkurt, "Clinical features and prognosis of herpetic anterior uveitis: a retrospective study of 111 cases," Int Ophthalmol, 30: 559-65, 2010.

[9] S. B. H. Katherine, Y. S. Ngim, J. Juliana, R. Norlina, "Herpes zoster keratouveitis with hypopyon and hyphema "Taiwan J Ophthalmol, 10(1):54-57, 2020.

[10] A. R. Suriada, L. M. Evelyn-Tai, L. K. Thavaratnam, I. Mohtar, W. H. Wan Hazabbah, "Report of a child with acute herpes zoster ophthalmicus induced partial third nerve palsy," Journal of acute diseases, 4:162-164, 2015.

[11] K. Othmana, L. M. Evelyn-Tai, M. N. Raja-Azmi, M. Julieanaa, A. J. Liza-Sharmini, J. Tharakan, I. Shatriah, "Concurrent hyphemia and orbital apex syndrome following herpes zoster ophthalmicus in a middle-aged lady," International Journal of Surgery Case Reports, 30:197-200, 2017.

[12] E. T. Jr. Cunningham, "Diagnosing and treating herpetic anterior uveitis," Ophthalmology, 107:2129-30, 2000.

[13] K. Babu, R. Kini, M. Philips, D. K. Subbakrishna, "Clinical profile of isolated viral anterior uveitis in a South Indian patient population," Ocul Immunol Inflamm, 22:356-9, 2014.

[14] S. K. H. Szeto, T. C. Y. Chan, R. L. M. Wong, A. L. K. Ng, E. Y. M. $\mathrm{Li}$, V. Jhanji, "Prevalence of Ocular Manifestations and Visual Outcomes in Patients with Herpes Zoster Ophthalmicus," Cornea, 36(3):338-342, 2017.

[15] R. Kahloun, S. Attia, B. Jelliti, A. Z. Attia, S. Khochtali, S. B. Yahia, and M. Khairallah, "Ocular involvement and visual outcome of herpes zoster ophthalmicus: review of 45 patients from Tunisia," North Africa. J Ophthal Inflamm Infect, 4:25, 2014.

[16] M. Hosogai, Y. Nakatani, K. Mimura, S. Mishi, H. Akiyama, "Genetic analysis of varicella-zoster virus in the aqueous humor in uveitis with severe hyphema," BMC Infectious Diseases, 17; 427, 2017. 